\title{
Novel BRAF mutation in melanoma: A case report
}

\author{
SERENA TRUBINI, ALESSANDRO UBIALI, CARLO TERENZIO PATIES and LUIGI CAVANNA
}

Onco-Hematologic Department, Ospedale Guglielmo da Saliceto, I-49-29121 Piacenza, Italy

Received June 27, 2017; Accepted November 17, 2017

DOI 10.3892/mco.2018.1555

\begin{abstract}
In melanoma, a number of specific genetic and genomic aberrations have been identified to be important in tumorigenesis. In particular, the mutant B-Raf proto-oncogene, Serine/Threonine kinase (BRAF) gene is the target of tailored therapy with kinase inhibitor molecules. Identification of the array of mutations in patients with melanoma will be useful in determining a genetic profile of the tumor with potential implications for treatment decisions. A rare aminoacidic insertion in codon 599 of the BRAF gene (c.1797_1798insACA, T599insT) was detected by using both direct (Sanger) sequencing and pyrosequencing techniques in a metastatic melanoma of a female elderly patient. As suggested in other clinical contexts including pilocytic astrocytoma, papillary thyroid carcinomas and anaplastic thyroid carcinomas, this unusual mutation may be associated with a modified spatial structure of activated P-loop, resulting in a constitutional activation of the BRAF protein. The patient died shortly following the test, thus no biological therapy was performed. Comparable data regarding treatment of melanoma patients with rare BRAF mutations is lacking, and the response to BRAF inhibitors requires further investigation.
\end{abstract}

\section{Introduction}

Mutations of BRAF oncogene are common in cutaneous melanomas, being found in as much as $50 \%$ of the total number of cases. Most mutations involve exon 15, exon 11 being interested in less than $1 \%$ of cases (1). V600E in exon 15 is by far the most common (2). This mutation modifies the spatial structure of activation P-loop, causing a 500-fold increase in BRAF kinase activity and facilitating the acquisition of secondary genetic events in cancer progression (3). Target therapy for cutaneous melanomas is focused on this mutation:

Correspondence to: Dr Serena Trubini, Onco-Hematologic Department, Ospedale Guglielmo da Saliceto, Piacenza, via G. Taverna, I-49-29121 Piacenza, Italy

E-mail: s.trubini@ausl.pc.it

Abbreviations: ATC, anaplastic thyroid carcinoma; PA, pilocytic astrocytoma; PTC, papillary thyroid carcinoma; TKI, tyrosine kinase inhibitor

Key words: BRAF, melanoma, rare mutations, sequencing, codon 599 small molecules (vemurafenib and dabrafenib) act as Tyrosine Kinase Inhibitors (TKIs), and interrupt the BRAF/MEK step in RAS/MEK/ERK pathway, inducing apoptosis in mutated cells (4). Less frequent BRAF mutations $(5,6)$ with alterations in the same crucial site, such as Threonine599 and Serine601 (7), have been described. Herein, we report a single case of primary cutaneous melanoma showing a mutation occurring in the P-loop activating site (c.1797_1798insACA, T599insT), which was not previously described in melanomas, but only rarely found in Pilocytic Astrocytoma (PA), Papillary Thyroid Carcinoma (PTC) and Anaplastic Thyroid Carcinoma (ATC) (8-11). In silico and in vitro data indicate that rare and/or complex mutations in codons 599-601 increase kinase activity similarly to the typical V600E $(8,9,12,13)$.

\section{Case report}

A 88-year-old female was presented with large, ulcerated superficial spreading melanoma on the left cheek, including a nodular polypoid component, widely ulcerated, with regression arising from a radial growth phase pigmented macula. Histologic examination showed a T4 lesion, Clark level V, with invasion into the subcutaneous fat (Fig. 1A). Angiolymphatic and perineural invasion were also described and mitotic rate of $20 / \mathrm{mm}^{2}$ was detected. Melanoma cells were epithelioid and spindle-shaped with marked pleomorphism. Sporadic monster cells were also observed (Fig. 1B-D). Specimen resulted strongly positive with $\mathrm{S}-100$ protein (4C4.9), anti-Melanosome (HMB-45) (Fig. 1D) and Melan-A (A103) immunostaining, weakly and focally for MiTF (C5/D5). Proliferative index, detected by Ki67 (30-9 clone), was $\sim 55 \%$. Abdominal ultrasound showed liver metastases. The patient died six months after diagnosis due to melanoma progression and a co-morbidity (renal failure). BRAF mutational status of the primary lesion was assessed by both Sanger sequencing and pyrosequencing, using the Anti-EGFR MoAb response BRAF Status kit (Diatech S.p.A., Jesi, Italy). Genetic analysis showed a rare aminoacidic insertion in codon 599 of the BRAF gene (1797_1798insACA, T599insT: Fig. 2) (GenBank NM_004333), resulting in a duplication of the same codon and an additional Threonine in the protein sequence (NCBI Ref Seq: NP_004324.2).

\section{Comment}

T599dup mutation was first described as associated to PA $(8,13,14)$, and eventually found in PTC and ATC (10-12). 
A
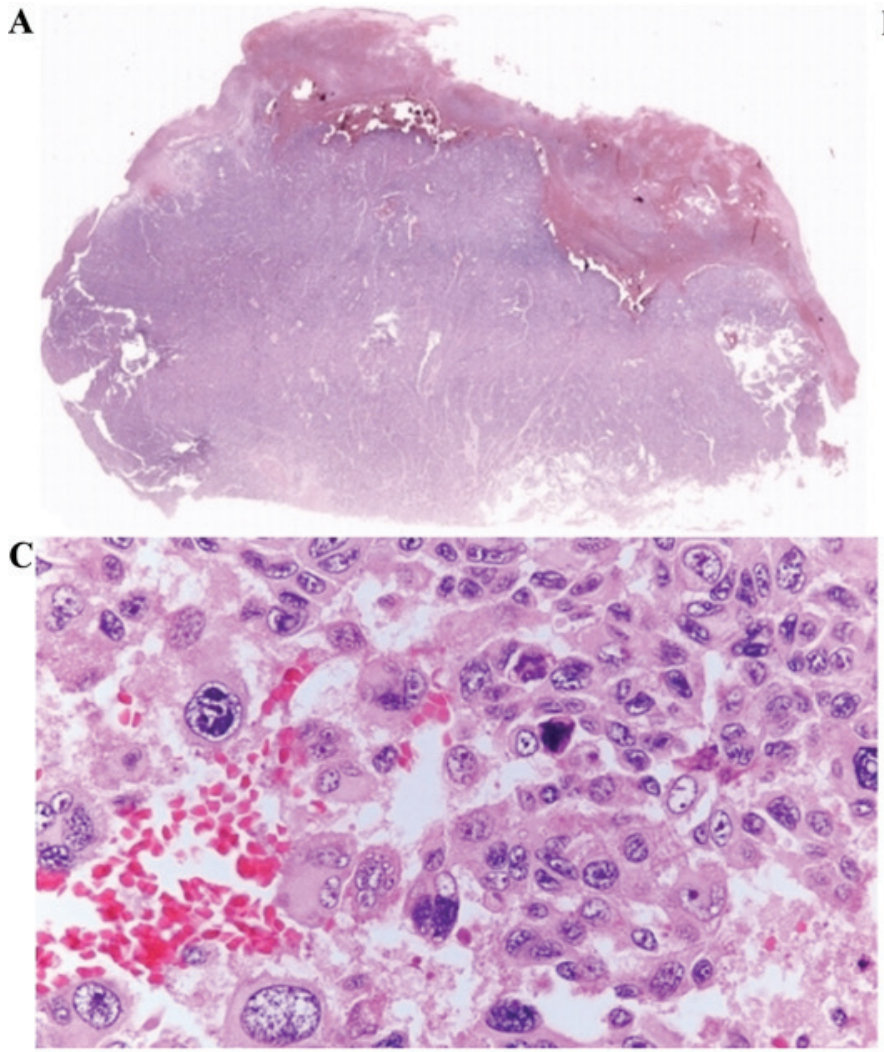

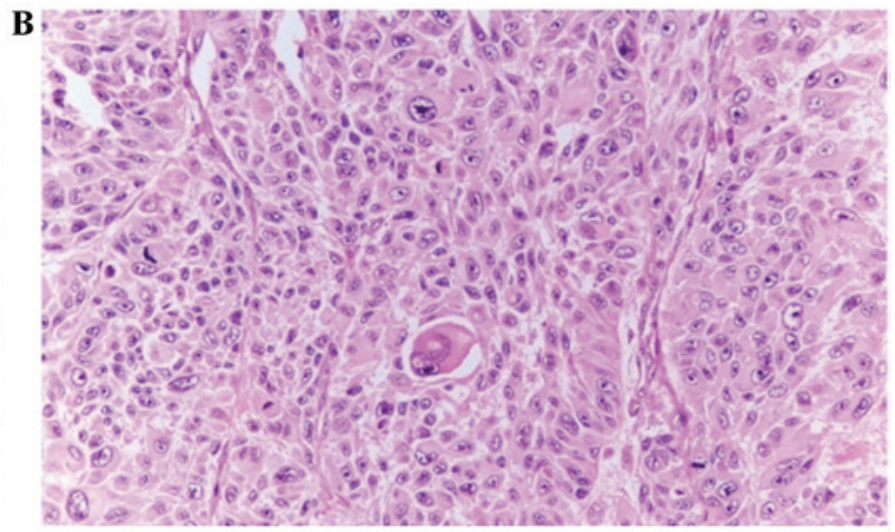

D

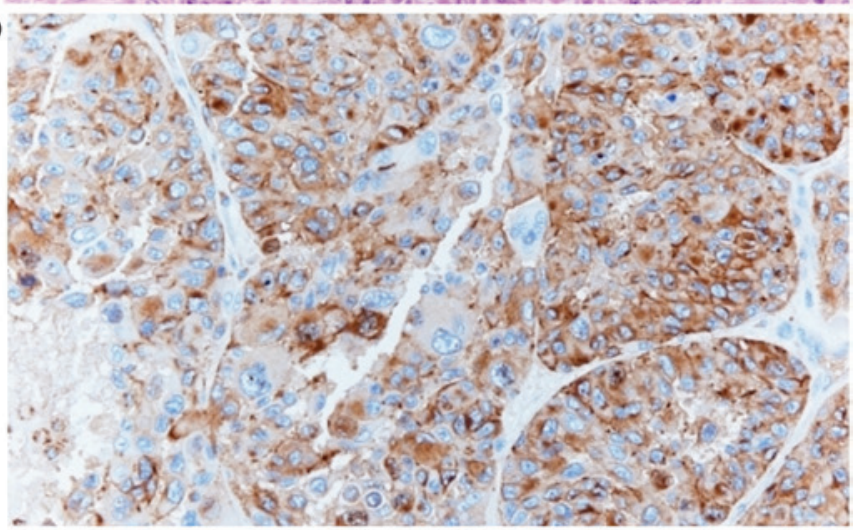

Figure 1. Histology. (A) A panoramic view (x2) of melanoma showing ulceration and extension into the subcutaneous fat. (B) Epithelioid melanoma cells with frequent mitotic figures and scattered monster cells (x20). (C) Higher magnification (x40) of pleomorphic epithelioid cells and isolated monster cells. (D) Diffuse immunostaining of melanoma cells for HMB45 (x40).

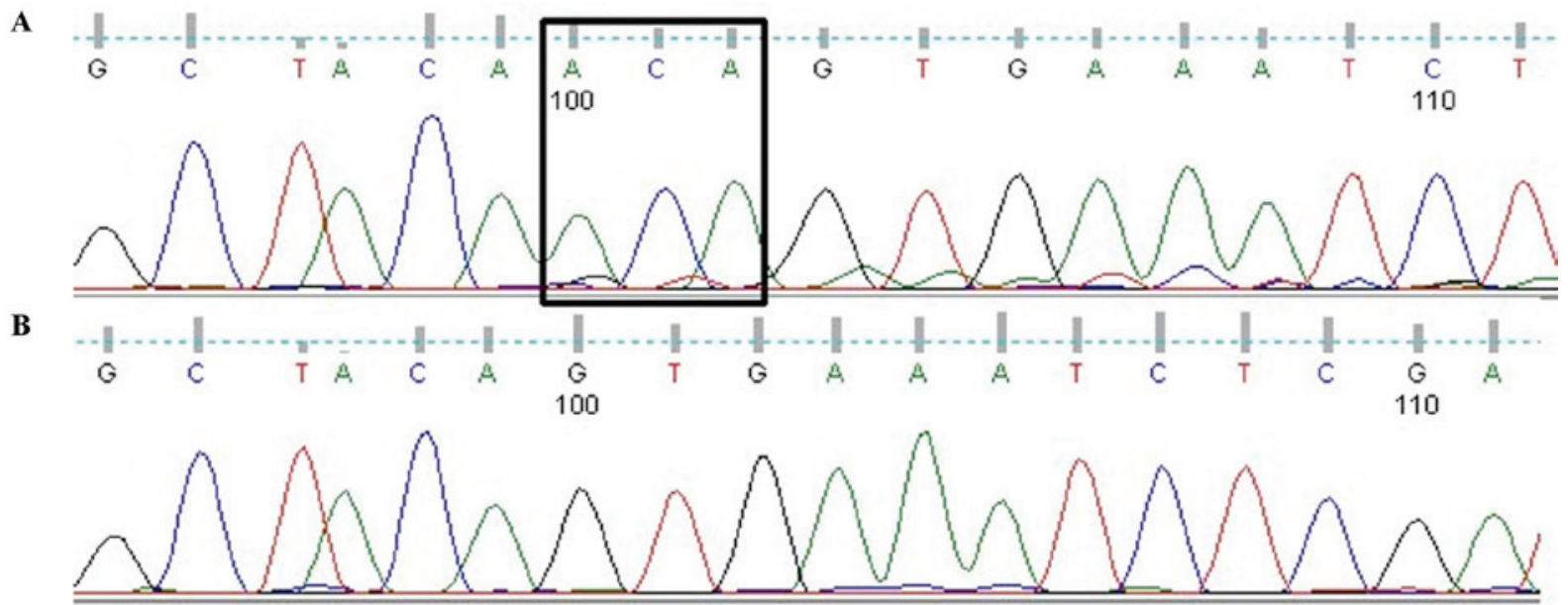

Figure 2. Direct sequencing electropherogram. (A) Mutation T599dup (insertion squared). (B) Wild-type sequence from Horizon DNA BRAF wild-type reference standard (FFPE Sections).

Although in this study and in cited reports $(8,10-14)$ all mutations lead to the same T599dup alteration at amino acid level, at DNA level they are described as an ACA duplication $(11,12)$, and as an insertion of TAC between $\mathrm{C}$ and $\mathrm{A}$ in the other studies. In silico analysis, performed on BRAFV599ins and BRAFT599insT $(9,12)$, indicates that mutations involving the P-loop make the active geometrical conformation more stable than the wild-type counterpart, causing an highly productive catalytic state. In vitro kinase assays carried out on BRAFV599ins and BRAFV600E (9) revealed a three-to-five-fold increase in the enzymatic activity (BRAF-induced phosphorylation of MEK and MAPK) of both mutants compared to BRAFWT. Similarly, evaluation of BRAFT599insT $(8,13)$ confirmed the tumorigenic potential of this mutation. The two cited TKIs are not currently employed in treatment of tumors other than advanced and metastatic melanoma. Their potential use in different clinical settings is being studied for lesions carrying the V600E mutation (clinical trial NCI-2013-01160), which is the target of TKIs. Thus, no data about the effectiveness of targeted therapy on 
rare $\mathrm{BRAF}$ mutations are available. In the described case, unfortunately the patient died only weeks after the genetic tests were performed. The potential response to BRAF inhibitors in presence of this mutation remains a matter for further studies.

\section{Competing interests}

The authors declare that they have no competing interests.

\section{References}

1. Greaves WO, Verma S, Patel KP, Davies MA, Barkoh BA Galbincea JM, Yao H, Lazar AJ, Aldape KD, Medeiros LJ and Luthra R: Frequency and spectrum of BRAF mutations in a retrospective, single-institution study of 1112 cases of melanoma. J Mol Diagn 15: 220-226, 2013.

2. Davies H, Bignell GR, Cox C, Stephens P, Edkins S, Clegg S, Teague J, Woffendin H, Garnett MJ, Bottomley W, et al: Mutations of the BRAF gene in human cancer. Nature 417: 949-954, 2002.

3. Wan PT, Garnett MJ, Roe SM, Lee S, Niculescu-Duvaz D, Good VM, Jones CM, Marshall CJ, Springer CJ, Barford D and Marais R; Cancer Genome Project: Mechanism of activation of the RAF-ERK signaling pathway by oncogenic mutations of B-RAF. Cell 116: 855-867, 2004.

4. Beck D, Niessner H, Smalley KS, Flaherty K, Paraiso KH, Busch C, Sinnberg T, Vasseur S, Iovanna JL, Drießen S, et al: Vemurafenib potently induces endoplasmic reticulum stress-mediated apoptosis in BRAFV600E melanoma cells. Sci Signal 6: ra7, 2013.

5. Long GV, Menzies AM, Nagrial AM, Haydu LE, Hamilton AL, Mann GJ, Hughes TM, Thompson JF, Scolyer RA and Kefford RF: Prognostic and clinicopathologic associations of oncogenic BRAF in metastatic melanoma. J Clin Oncol 29: 1239-1246, 2011.

6. Heinzerling L, Kühnapfel S, Meckbach D, Baiter M, Kaempgen E, Keikavoussi P, Schuler G, Agaimy A, Bauer J, Hartmann A, et al: Rare BRAF mutations in melanoma patients: Implications for molecular testing in clinical practice. Br J Cancer 108: 2164-2171, 2013.
7. Dhillon AS and Kolch W: Oncogenic B-Raf mutations: Crystal clear at last. Cancer Cell 5: 303-304, 2004.

8. Jones DT, Kocialkowski S, Liu L, Pearson DM, Ichimura K and Collins VP: Oncogenic RAF1 rearrangement and a novel BRAF mutation as alternatives to KIAA1549: BRAF fusion in activating the MAPK pathway in pilocytic astrocytoma. Oncogene 28: 2119-2123, 2009.

9. Moretti S, Macchiarulo A, De Falco V, Avenia N, Barbi F Carta C, Cavaliere A, Melillo RM, Passeri L, Santeusanio F, et al: Biochemical and molecular characterization of the novel BRAF(V599Ins) mutation detected in a classic papillary thyroid carcinoma. Oncogene 25: 4235-4240, 2006.

10. Schulten HJ, Salama S, Al-Mansouri Z, Alotibi R, Al-Ghamdi K, Al-Hamour OA, Sayadi H, Al-Aradati H, Al-Johari A, Huwait E, et al: BRAF mutations in thyroid tumors from an ethnically diverse group. Hered Cancer Clin Pract 10: 10, 2012.

11. Gauchotte G, Philippe C, Lacomme S, Léotard B, Wissler MP, Allou L, Toussaint B, Klein M, Vignaud JM and Bressenot A: BRAF, p53 and SOX2 in anaplastic thyroid carcinoma: Evidence for multistep carcinogenesis. Pathology 43: 447-452, 2011.

12. Barollo S, Pezzani R, Cristiani A, Redaelli M, Zambonin L, Rubin B, Bertazza L, Zane M, Mucignat-Caretta C, Bulfone A, et al: Prevalence, tumorigenic role, and biochemical implications of rare BRAF alterations. Thyroid 24: 809-819, 2014.

13. Eisenhardt AE, Olbrich H, Röring M, Janzarik W, Anh TN, Cin H, Remke M, Witt H, Korshunov A, Pfister SM, et al: Functional characterization of a BRAF insertion mutant associated with pilocytic astrocytoma. Int J Cancer 129: 2297-2303, 2011.

14. Yu J, Deshmukh H, Gutmann RJ, Emnett RJ, Rodriguez FJ, Watson MA, Nagarajan R and Gutmann DH: Alterations of BRAF and HIPK2 loci predominate in sporadic pilocytic astrocytoma. Neurology 73: 1526-1531, 2009. 